\title{
A morphometric analysis of the infant calvarium and dura
}

\author{
Eric Breisch • Elisabeth A. Haas • \\ Homeyra Masoumi · Amy E. Chadwick • \\ Henry F. Krous
}

Accepted: 16 December 2009/Published online: 20 January 2010

(C) The Author(s) 2010. This article is published with open access at Springerlink.com

\begin{abstract}
Literature addressing the anatomic development of the dura and calvarium during childhood is limited. Nevertheless, histological features of a subdural neomembrane (NM), including its thickness and vascularity, developing in response to an acute subdural hematoma (SDH) have been compared to the dura of adults to estimate when an injury occurred. Therefore, we measured the morphometric growth of the calvarium and dura and the vascular density within the dura during infancy. The mean thicknesses of the calvarium and dura as a function of occipitofrontal circumference (OFC), as well as the mean number of vessels per $25 \times$ field, were determined from the right parasagittal midparietal bone lateral to the sagittal suture of 128 infants without a history of head trauma. Our results showed that as OFC increased, the mean thicknesses of the calvarium and dura increased while the vascular density within the dura decreased. Our morphometric data may assist in the interpretation of subdural NM occurring during infancy. We recommend future investigations to confirm and extend our present data, especially by evaluating cases during later infancy and beyond as well as by sampling other anatomic sites from the calvarium. We also
\end{abstract}

E. Breisch - E. A. Haas - H. Masoumi - A. E. Chadwick ·

H. F. Krous ( $\square)$

Department of Pathology, Rady Children's Hospital and Health Center, 3020 Children's Way, MC5007, San Diego, CA 92123, USA

e-mail: hkrous@rchsd.org

E. Breisch

Department of Surgery/Division of Anatomy, UCSD School of Medicine, La Jolla, CA, USA

H. F. Krous

Departments of Pathology \& Pediatrics, UCSD School

of Medicine, La Jolla, CA, USA recommend morphometric evaluation of subdural NM associated with SDH in infancy and childhood.

Keywords Dura - Calvarium · Infant · Anatomy · Vessel $\cdot$ Neomembrane $\cdot$ Forensic

\section{Introduction}

A subdural hematoma ( $\mathrm{SDH}$ ) is an important complication of abusive or accidental head trauma in infants and children. The organization of a SDH involves formation of a neomembrane (NM), the histological features of which include fibroblastic and vascular proliferation, collagen formation, and hemosiderin deposition. The appearance and thickness of a NM have been compared to the underlying normal dura in an attempt to estimate its histological age, thereby determining when an injury was sustained. The aging of NMs in infants and children has been based on observations of NMs occurring in adults that were reported in 1936 by Munro and Merritt [1] and tabulated in both editions of Forensic Neuropathology authored by Leetsma [2,3]. It is unclear whether the observations of Munro and Merritt [1] are valid in early childhood, given that no similar studies have been undertaken in this age period. Even the literature regarding the developmental anatomy of the calvarium and dura in early childhood is limited. One study of grossly normal dura from 11 children found it was "relatively vascular", occasionally revealed hemosiderin, and nearly always contained intradural blood [4]. Other investigators reported that $31 \%$ of SIDS cases and $13 \%$ of control infants had organizing subdural neomembranes, a difference that was not statistically significant [5]. In all but two cases, birth trauma was excluded. Recent studies have provided elegant qualitative 
descriptions of the meningeal embryology and anatomy of the dura, but morphometric data were not included [6-8]. Therefore, the aim of this study is to report the morphometric development of the calvarium and dura in infants.

\section{Methods}

This study was approved by the Rady Children's HospitalSan Diego (RCHSD) Institutional Review Board.

Cases without evidence of head trauma were selected from the San Diego SIDS Research Project database. Standardized perpendicular microscopic sections of the sagittal suture and adjacent right parasagittal mid-parietal bone with attached dura were obtained prospectively from 132 infants who died suddenly and underwent autopsy at either the San Diego Medical Examiner's Office or RCHSD. Four cases were excluded: two cases without documented OFC at death, and two because of excessive tangential orientation. In 11 cases, the calvarium had been stripped from the dura; these cases are included only in the calvarium measurements, but excluded from the dural and vessel calculations. After rapid decalcification of the paraffin block with 5\% hydrochloric acid, microscopic sections were cut at $4 \mu \mathrm{m}$ and stained with hematoxylin and eosin. This method allows excellent histological preservation of the tissues (and is routinely used for the interpretation of pediatric bone marrow biopsies in our department). Immunohistochemical staining was not undertaken to identify endothelium.

A diagnosis of SIDS was assigned according to the criteria for the general definition recently proposed in 2004 in San Diego [9]. Diagnoses of non-SIDS cases were based upon analysis of information obtained from the medical history, circumstances of death, and postmortem findings.

All measurements were made directly through the microscope using a micrometer. Mean thicknesses of the parietal bone of the calvarium and the attached dura were determined for each case from measurements of three contiguous $25 \times$ power fields $(10 \times \mathrm{pf}) 2.5 \mathrm{~cm}$ lateral to the sagittal suture. One measurement was obtained in each contiguous field. Measurements were not taken from sites showing mild, microfocal acute hemorrhage that occurred in a small percentage of the specimens. The mean vascular density for each case was calculated after counting all of the blood vessels in five random $25 \times$ power fields of dura beginning approximately $1 \mathrm{~cm}$ and extending laterally from the superior sagittal suture. The mean number of blood vessels per $25 \times$ power field rather than per square millimeter was chosen since the thin membranous nature of the specimens being measured often did not fill the entire microscopic field. Calculation of vascular density within the dura using square millimeters as the denominator would have resulted in misleading data, given that some of the microscopic fields were not completely filled by tissue. Capillaries were the predominate vessels in each of the sections. Mean vascular densities were not calculated specifically for the periosteal and subdural sides of the dura. Each of the calculated means was plotted as a function of OFC.

\section{Statistical analyses}

The difference in distribution of measurements by quartile groups of age at death was calculated by Analysis of Variance; these statistics were performed using SPSS version 11.5. A Simple Interactive Statistical Analysis (SISA) procedure was used to test statistical significance of a difference between two correlation coefficients from a single sample.

\section{Results}

The 128 infants were classified as follows: SIDS, 61 (48\%); other natural deaths, 36 (28\%); unclassified sudden infant death (USID) [9], $22(17 \%)$; and accidents not involving head trauma, 9 (7\%). The mean \pm standard deviation (SD) for age was $84.5 \pm 72$ days, and the range was $0-345$ days. Among the 128 infants, $49 \%$ were white, $31 \%$ were Hispanic, 9\% were African American, $7 \%$ were Asian/American Indian/Pacific Islander, $2 \%$ were other, and $2 \%$ were unknown. Analysis of ethnicity data revealed the two infants with mixed race heritage had the thickest calvarium; there were no other significant results. Males comprised $63 \%$ of the sample. Among 107 cases with available information, $60 \%$ were born at term gestation. The OFC was within the normal range for postconceptual age for all of the cases. None of the cases had evidence of microcephaly, macrocephaly or hydrocephalus.

The thicknesses of the calvarium $(P<0.01)$ and dura $(P<0.01)$ increase with increasing OFC (Figs. 1, 2). The difference between slopes of the growth of the calvarium and the dura is statistically significant at the 0.05 level. Conversely, vascular density within the dura decreases with increasing OFC $(P<0.05)$ (Fig. 3).

Tables 1,2, 3 show the medians and percentiles of the measured variables by age quartile during infancy at the time of death. The median thickness of the calvarium during the first quartile was $1037 \mu \mathrm{m}$ compared to $1689 \mu \mathrm{m}$ during the third quartile. The median thickness of the dura during the first quartile was $485 \mu \mathrm{m}$ compared to $607 \mu \mathrm{m}$ during the third quartile. The differences of the thickness of the calvarium and dura were statistically significant between groups $(P<0.01)$. The median number of vessels per 


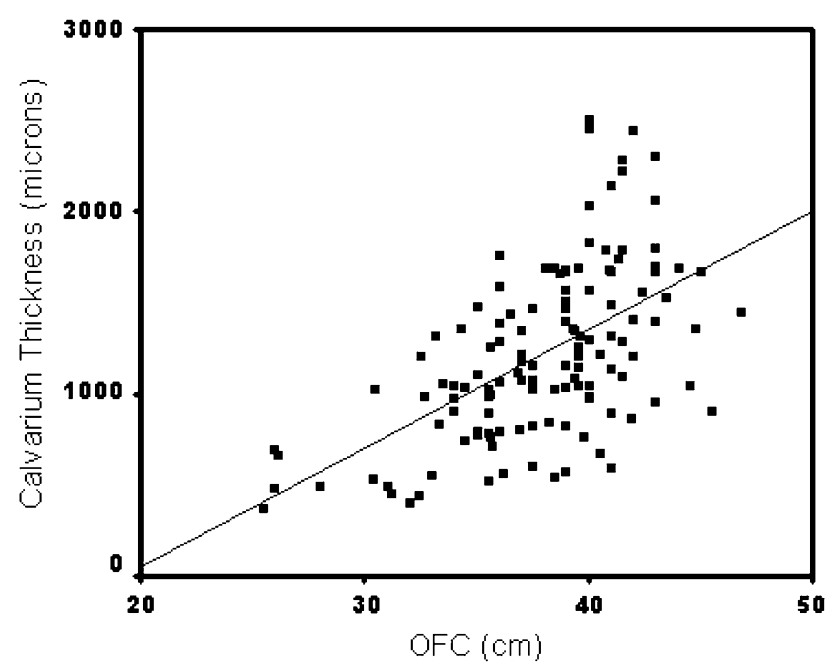

Fig. 1 The thickness of the calvarium increases with increasing OFC. Pearson correlation coefficient $=.569, \quad r^{2}=.324, \quad P<0.01$. $(n=128)$

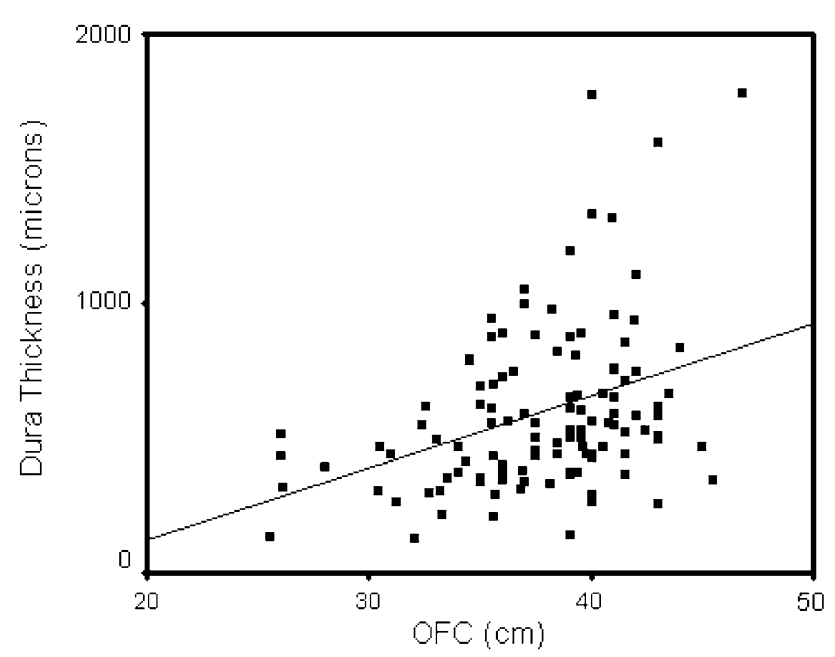

Fig. 2 The dura increases in thickness with increasing OFC in infancy. Pearson correlation coefficient $=.370, r^{2}=.137, P<0.01$. $(n=128)$

$25 \times$ power field ranged between 3.2 and 4.2 during the four quartiles of infancy, but the differences were not statistically significant. Figure 4a through c illustrate the histology of the calvarium and dura at OFCs of 26, 33, and $40 \mathrm{~cm}$, respectively.

\section{Discussion}

Our data, obtained from prospectively collected autopsy specimens from infants between the ages of 0-345 days, provide baseline measurements of bone and dura thickness as a function of OFC. We observed a progressive increase

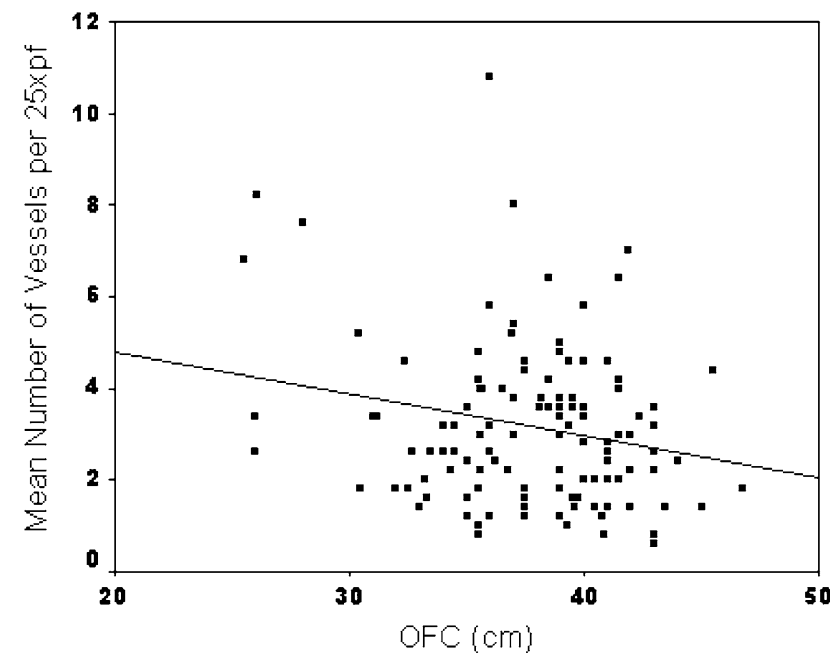

Fig. 3 The density of vessels in the dura gradually decreases with increasing OFC during infancy. Pearson correlation coefficient is $-.218, r^{2}=.0474, P<0.05 .(n=117)$

Table 1 Calvarium thickness in microns by age and percentile

\begin{tabular}{lccclc}
\hline $\begin{array}{l}\text { Age } \\
\text { days })\end{array}$ & Number & $\begin{array}{l}\text { 25th } \\
\text { percentile }\end{array}$ & Median & $\begin{array}{l}\text { 75th } \\
\text { percentile }\end{array}$ & $P$ value \\
\hline $0-90$ & 81 & 771 & 1037 & 1320 & $<.01$ \\
$91-180$ & 32 & 1104 & 1455 & 1779 & \\
$181-270$ & 11 & 1397 & 1689 & 1799 & \\
$271-365$ & 4 & 1018 & 1463 & 1640 & \\
\hline
\end{tabular}

Table 2 Dural thickness in microns by age and percentile

\begin{tabular}{llllcl}
\hline $\begin{array}{l}\text { Age } \\
\text { (days) }\end{array}$ & Number & $\begin{array}{l}\text { 25th } \\
\text { percentile }\end{array}$ & Median & $\begin{array}{l}\text { 75th } \\
\text { percentile }\end{array}$ & $P$ value \\
\hline $0-90$ & 77 & 354 & 485 & 652 & $<.01$ \\
$91-180$ & 27 & 530 & 663 & 858 & \\
$181-270$ & 10 & 467 & 607 & 1029 & \\
$271-365$ & 3 & 347 & 469 & 530 & \\
\hline
\end{tabular}

Table 3 Number of dural vessels per $25 \times$ power field by age and percentile

\begin{tabular}{llllll}
\hline $\begin{array}{l}\text { Age } \\
\text { (days) }\end{array}$ & Number & $\begin{array}{l}25 \text { th } \\
\text { percentile }\end{array}$ & Median & $\begin{array}{l}\text { 75th } \\
\text { percentile }\end{array}$ & $P$ value \\
\hline $0-90$ & 77 & 1.9 & 3.2 & 4.2 & NS* \\
$91-180$ & 27 & 1.4 & 2.6 & 3.6 & \\
$181-270$ & 10 & 2.1 & 2.6 & 3.45 & \\
$271-365$ & 3 & 1.4 & 3.4 & 4.4 & \\
\hline
\end{tabular}

* Not significant

in the thickness of the calvarium with increasing OFC (Figs. 1, 4a-c). The postnatal growth of the calvarium proceeds very rapidly during the first year and is followed 

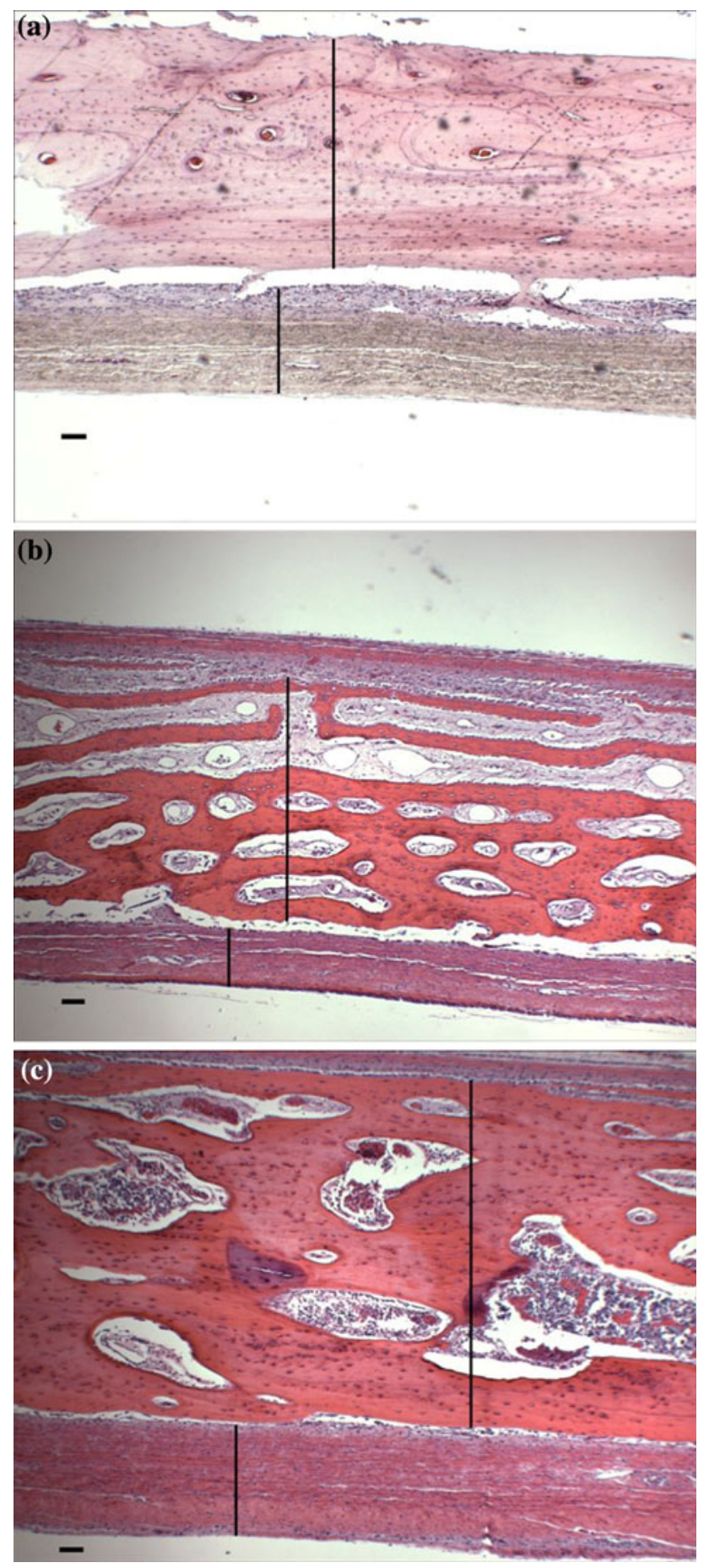

Fig. 4 a The calvarium surfaces the dura of an infant with an OFC of $26 \mathrm{~cm}$. The vertical bars indicate dimensions selected for measuring the thickness of the calvarium and dura. The horizontal marker equals $100 \mu \mathrm{m}$. Hematoxylin and eosin, $40 \times$. b The calvarium surfaces the dura of an infant with an OFC of $33 \mathrm{~cm}$. The vertical bars indicate dimensions selected for measuring the thickness of the calvarium and dura. The horizontal marker equals $100 \mu \mathrm{m}$. Hematoxylin and eosin, $40 \times$. $\mathrm{c}$ The calvarium surfaces the dura of an infant with an OFC of $40 \mathrm{~cm}$. The vertical bars indicate dimensions selected for measuring the thickness of the calvarium and dura. The horizontal marker equals $100 \mu \mathrm{m}$. Hematoxylin and eosin, $40 \times$ by a much slower growth rate until approximately the seventh year when the skull approaches adult size [10]. Since our cases were limited to infants, we are unable to address growth during the second year of life.

Expansional growth of the calvarium during the first two years of life is achieved by membranous ossification at the sutures and along the outer and inner surfaces in a process characterized by concomitant accretion and absorption of the deep and superficial surfaces, thus permitting growth accommodation for changes in the curvature of the calvarium [11]. Previous reports indicate that the calvarium is unilaminar until the diploe appear in approximately the fourth year of life, forming a trilaminar calvarium [10] In an adult, the inner table of the calvarium is thinner than the outer table, which accounts for the increased incidence of inner table fracture compared to the outer table in head trauma [10, 12]. In our analysis, we were unable to establish when that disparity in thickness develops, since the calvarium remained unilaminar throughout the study interval.

The normal dura consists of outer and inner layers [13]. The outer layer is a very dense fibrous membrane that is tightly attached to the inner table of the calvarium and serves as its periosteum. The inner layer is comprised of less dense fibrous connective tissue lined on its inner aspect by a layer of mesothelial cells that are in direct contact with the arachnoid layer of the leptomeninges [10]. Our study restricted the measurement of thickness of the dura to the dense components of the inner and outer layers since mechanical forces required for removal of the calvarium routinely disrupted the mesothelial layer. We found that the thickness of dura increased with increasing OFC (Figs. 2, $4 \mathrm{a}-\mathrm{c})$, but at a slower rate when compared with the rate of increase of the thickness of the calvarium (Pearson correlation coefficients are .370 and .569 , respectively; the difference between the slopes of the growth of the calvarium and the dura is significant at the 0.05 level).

The blood supply to the dura is derived from the meningeal vessels. The vascular pattern of the inner and outer dura have been described in adults [6, 14], but not in early childhood to our knowledge. In our specimens, the small arterioles and venules were located primarily in the dense collagenous part of the dura while the capillaries were in the periosteum. We are unaware of previous studies in which the density of blood vessels in the dura was measured. Our data show that there is a slight decrease in the vascular density with increasing head circumference (Fig. 3). The significance of this finding is unknown, but it suggests a possible decrease in metabolic demand despite increasing thickness. The mere presence of blood vessels in the dura in the absence of other histological features of NMs must be viewed from a forensic viewpoint as normal 
in that it provides nourishment to this developing structure. Depending on their age, NMs following SDH require the presence of other histological findings such as fibrin, siderophages, fibroblastic proliferation, and/or collagen deposition.

In practice and as expert witnesses in cases of pediatric abusive head injury, pathologists commonly use data reported by Munro and Merritt in 1936 [1] to "age" neomembranes evolving from acute subdural hematomas. The Munro and Merritt data were derived from adults found to have subdural neomembranes at autopsy. To our knowledge, it has not been established that their data (which have been tabulated by Leestma [2]) are valid in infants and toddlers; nor did they take into account the morphometric development of the calvarium and dura. The recent study examining microscopic features of grossly normal dura from one third trimester intrauterine death and 10 infants between 2 weeks and 10 months of age provided data on the frequency of intradural blood and intra- and extracellular hemosiderin, but not on quantitative changes in dural thickness and vascularity or changes in the appearance of the calvarium, as undertaken in our study [4]. These investigators did emphasize, however, the importance of being familiar with the pediatric dura in order to properly interpret true pathologic findings when they are present.

Our study is limited by restricting measurements to the right mid-parietal bone and dura taken approximately $2.5 \mathrm{~cm}$ lateral to the sagittal suture. The calvarium gets progressively thicker as the lateral distance from the sagittal suture increases until a rather uniform thickness is achieved as the base of the skull is approached. Thus, our data can not be generalized to all sites within the cranium. In this regard, fractures (and NMs) have been observed in all regions of the cranium. The susceptibility of the calvarium to fracture is influenced by not only the direction and force of the impact, but also by the unique anatomic characteristics and biomechanical properties at each site. Secondly, our sample contains relatively few cases from the last three months (quartile) of infancy and none after the first birthday.

However, our study is strengthened by the large number of cases that were collected prospectively, use of standardized microscopic sections, and basing the results on OFC rather than gestational or postnatal age, body length, or body weight. When our measurements were analyzed with respect to the latter variables, the results produced a scattergram lacking any developmental interpretation. Finally, our results provide morphometric data for future reference and comparison.

In conclusion, we have provided morphometric data on the growth of the calvarium, dura, and density of dural vessels during infancy which can serve as a baseline for further investigation of NMs after subdural hemorrhage in infancy. These data may also be useful in the morphometric evaluation of other disorders, including bone responses of the infant calvarium to biomechanical stresses and craniofacial anomalies. We recommend future investigations to confirm our findings and to extend our present data by not only evaluating cases during later infancy and beyond but also by sampling other anatomic sites from the calvarium. Finally, an analysis of NMs as a function of the developmental status of the calvarium and dura is indicated.

\section{Key points}

1. The histological features of neomembranes, a reparative response to acute subdural hematomas, have been used to age their duration from the time of an injury in early childhood.

2. Efforts to age neomembranes have been based on data derived from characteristics of the dura in adults, but not early childhood.

3. Although qualitative descriptions exist, quantitative morphometric analyses of the calvarium and dura in early childhood are lacking.

4. Our data show that as OFC increased in infancy, the mean thicknesses of the calvarium and dura increased while the vascular density within the dura decreased.

Acknowledgments The cooperation of the forensic pathologists at the San Diego County Medical Examiner's Office is greatly appreciated.

Open Access This article is distributed under the terms of the Creative Commons Attribution Noncommercial License which permits any noncommercial use, distribution, and reproduction in any medium, provided the original author(s) and source are credited.

\section{References}

1. Munro D, Merritt HH. Surgical pathology of subdural hematoma. Based on a study of one hundred and five cases. Arch Neurol Psychiatry. 1936;35:64-78.

2. Leestma JE. Forensic neuropathology. New York: Raven Press; 1988.

3. Leestma JE. Forensic neuropathology. 2nd ed. Boca Raton, FL: CRC Press; 2009.

4. Croft PR, Reichard RR. Microscopic examination of grossly unremarkable pediatric dura mater. Am J Forensic Med Pathol. 2009;30:10-3.

5. Rogers CB, Itabashi HH, Tomiyasu U, Heuser ET. Subdural neomembranes and sudden infant death syndrome. J Forensic Sci. 1998;43:375-6.

6. Kerber CW, Newton TH. The macro and microvasculature of the dura mater. Neuroradiology. 1973;6:175-9.

7. Mack J, Squier W, Eastman JT. Anatomy and development of the meninges: implications for subdural collections and CSF circulation. Pediatr Radiol. 2009;39:200-10. 
8. Squier W, Mack J. The neuropathology of infant subdural haemorrhage. Forensic Sci Int. 2009;187:6-13.

9. Krous HF, Beckwith JB, Byard RW, et al. Sudden infant death syndrome and unclassified sudden infant deaths: a definitional and diagnostic approach. Pediatrics. 2004;114:234-8.

10. Williams PL, Warwick R. Gray's anatomy. 36th British Edition. Philadelphia: W.B. Saunders Co/Churchill Livingstone; 1980.
11. Ford EHR. The growth of the foetal skull. J Anat. 1956;90:63-72.

12. Hollinshead WH. Anatomy for Surgeons. Head and Neck. vol 1. 3rd ed. Harper \& Row; 1982. p. 4-5.

13. Carpenter MB. Human neuroanatomy. 7th ed. Baltimore: Williams and Wilkins Co; 1981.

14. Lang J. Zur vascularisation der Dura mater cerebr. I. Z Anat Entwickl-Gesch. 1971;135:20-34. 